\title{
An Ethical Approach to Decision Design
}

\author{
Marion G. Ben-Jacob \\ Department of Mathematics and Computer Sciences, Mercy College, Dobbs Ferry, New York \\ Email: mbenjacob@mercy.edu
}

How to cite this paper: Ben-Jacob, M.G. (2021) An Ethical Approach to Decision Design. Open Journal of Applied Sciences, 11, 665-671.

https://doi.org/10.4236/ojapps.2021.116048

Received: May 20, 2021

Accepted: June 21, 2021

Published: June 24, 2021

Copyright (c) 2021 by author(s) and Scientific Research Publishing Inc. This work is licensed under the Creative Commons Attribution International License (CC BY 4.0).

http://creativecommons.org/licenses/by/4.0/

\begin{abstract}
This paper describes an approach to ethical decision design. It discusses the use of several mathematical tools applicable to the world of economics and business. It then demonstrates how the tools analogously extend themselves to making ethical decisions as supported by personal values. A review of the mathematics, including the design of a decision tree, the concept of mathematical expectation, and mathematical modeling are included.
\end{abstract}

\section{Keywords}

Design, Ethics, Business, Decision Trees, Expected Value, Mathematical Models

\section{Introduction}

This paper uses mathematical tools to analyze both business and other ethical situations. An overriding mathematical concept, be it in the business world or the ethics world, is the idea of a mathematical model. This entails making certain assumptions or accepting hypothesis and analyzing the problem based on these. Of course, the result of the analysis is not guaranteed to be feasible or acceptable. The procedure then would retrace itself, adjusting the original hypotheses.

A decision tree is a predictive model. The predictions are made based on a series of determinations which can be objective and mathematically based or subjective depending on the circumstances. This tool is useful to show the routes by which the various possible outcomes can be obtained. There are basically two types of decision trees, 1) classification trees which are used to classify or divide the given information into predefined categories of a target variable and 2) regression trees when the target variable is numeric. In both these cases, the graphic representation of the issue aids in reaching a conclusion. Usually, the latter type of tree is used in business and economics as there is a monetary concern involved [1]. We purport that a decision tree is applicable in the determination of 
ethical pursuits according to accepted values and will so demonstrate via example.

Expected value is a numeric computation that predicts a value of a variable, by summing all possible values each multiplied by the probability of its occurrence. It is like a weighted average [1].

\section{The Mathematics}

A decision tree is sometimes called a classification tree. It is generated by nodes and arcs, the beginning node or the root is displayed on the far left. Adecision tree is pictorial toolin which each internal (non-leaf) node is labeled with an input feature. The arcs emanating from a node labeled with a feature demonstrate the paths or outcomes that may occur after that input feature is accepted. When a path ends, that final node is called a leaf of the tree [1].

There are four major steps in the process of decision analysis. They are the following:

1) Decision structuring: identifying the alternatives, uncertainties that affect the outcome, and the criteria for choosing among the competing alternatives.

2) Assessment and information gathering: assessing the probabilities of the uncertain events and assigning values to the consequences of a decision.

3) Evaluation of the decision problem: analyzing the information in steps $i$ and ii to determine which alternatives to undertake.

4) Sensitivity analysis: determining if the decision is sensitive to changes in probabilities, valuation of outcomes, or other assumptions made by the decision maker [2].

In business and economic models, decisions are usually based on maximizing net cash flow and are based on the Expected Monetary Value (EMV) of alternatives. Expected Value (EV) is a mathematical concept found in probability theory, and EMV is its application when the value is a monetary one. It may be defined as the sum of the outcomes multiplied by their probabilities and is determined by multiplying each outcome by its probability and then summing these products. Thus,

$$
E(x)=\mu=\sum x f(x)
$$

where $x$ is a discrete random variable and $f(x)$ its probability function [1].

As an example, consider, a car dealership, for which sales data reveal that, for the past 300 days of operation, there were 30 days when it sold no cars, 60 days when it sold 1 car, 84 days when it sold 2 cars, 72 days when it sold 3 cars, and 54 days when it sold 4 cars. The discrete random variable, $x$, is the number of cars sold during a day. From historical data we know that it can assume a value from 0 to 4 . From historical data we also know the probabilities of selling $0,1,2$, 3 , or 4 cars on a given day-namely, $30 / 300$ or $0.10 ; 60 / 300$ or $0.20 ; 84 / 300$ or $0.28 ; 72 / 300$ or $0.24 ; 54 / 300$ or 0.54 . To determine the Expected Value of cars sold in a day, one multiplies the values by their respective probabilities and adds these products. 
The Expected Value can thus be represented by the following Table:

\begin{tabular}{ccc}
\hline$x$ & $f(x)$ & $x f(x)$ \\
0 & 0.10 & 0 \\
1 & 0.20 & 0.2 \\
2 & 0.28 & 0.56 \\
3 & 0.24 & 0.72 \\
& 0.18 & 0.72 \\
4 & & 2.2 EV That is, the expected value of \\
& cars can sell in a day.
\end{tabular}

This of course leads us to believe that our actions both in the business world and our ethical behavior is more consequent based than we may initially recognize. Our actions are often dictated by the outcome of the behavior as opposed to the principles that guided the behavior. This idea will be expounded upon in the sections on ethics.

\section{An Example from the Business World}

The Benglaze company manufactures two types of widgets, widget $B$ and widget G. They are considering three possible choices for the company. Choice 1) is to improve both widgets which might produces a payout in terms of increased revenue net of costs, but they would have to invest $\$ 1,000,000$. A mathematical model was generated based on the research. The research was done and came back with a $30 \%$ chance of $\$ 200,000$ - but a $70 \%$ chance that it would by only $\$ 600,000$ based on this mathematics model.

Another choice the company is considering is to improve only widget G. This would cost only $\$ 400,000$ as an investment but research again shows that there is a $20 \%$ chance of a payout of $\$ 100,000$ but an $80 \%$ chance of it being only $\$ 400,000$.

The third choice is of course, to do nothing. This option requires no investment but has no projected payout.

The Expected Value of the return of the first choice is $0.30(2,000,000)+0.70$ $(600,000)=600,000+420,000=\$ 1,020,000$.

To realize a profit, they would have to subtract the investment of $\$ 1,000,000$ leaving $\$ 20,000$.

The Expected Value of the return of the second choice is $0.20(1,000,000)+$ $0.80(400,000)=200,000+320,000=\$ 520,000$. Once again to realize the profit one need to subtract the cost of the investment and the results are $\$ 520,000-$ $400,000=\$ 120,000$.

The use of a decision tree would look like this. From the root, three branches, would emanate, each one representing one of the options. Figure 1 is an example of a business decision tree. 


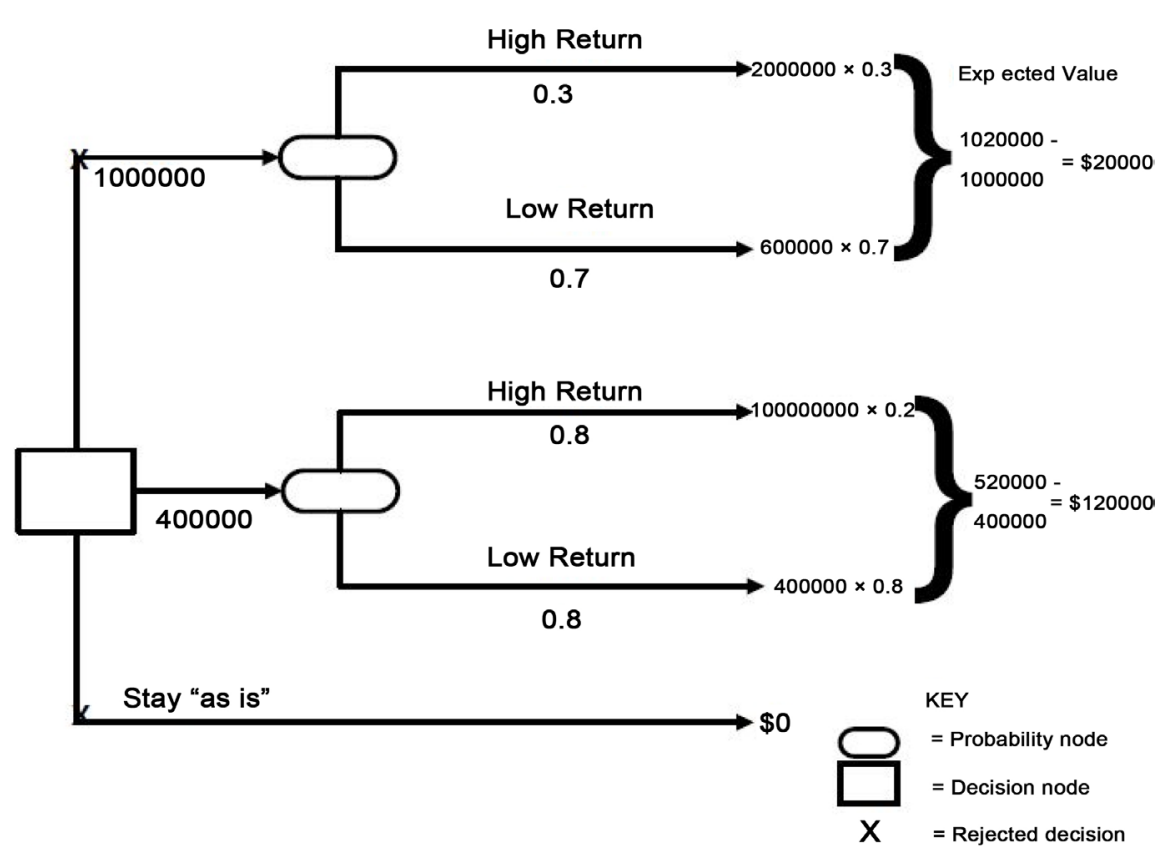

Figure 1. Example of a business decision tree [3].

\section{An Examples of Moral Principles or Personal Values}

We contend that the aforementioned tools can be modified and applied to making ethical decision in life and will demonstrate this via an example.

Consider the ethical dilemma based on the work of Glasser [4].

The administrative assistant, who has been with the organization for more than fifteen years, has been involved in a car accident that has caused her to lose the use of her right hand. She can no longer type or effectively perform many of the duties she had done previously. The organization, which is rather small, has a tight budget and does not have sufficient funds to pay for an additional administrative assistant. The injured administrative assistant has such a strong connection to the organization, as does the organization to her, that she would like to remain at the organization. Moreover, it is unlikely that she could find other employment at this time.

Let us suppose that after an extended medical leave with pay it is determined that the administrative assistant's injury is irreversible and that she will never regain full control of her hand. Let us suppose further that with the use of her left hand alone she will never be able to perform more than $10 \%$ of her previous duties. The stakeholders involved include the executive director, the leadership or board of directors of the organization, and the administrative assistant, among others. The preference of each of the stakeholders now is whether to discontinue her employment (terminate her) or to keep her on. Keeping her on could be handled in any number of ways, including restructuring her position and pay while finding another person to replace her previous duties, or simply keeping her on and tolerating the inefficiency of her performance; however, this is resolved, let us assume that the decision to keep her on is more costly to the or- 
ganization, then simply terminating her and finding a competent replacement. From an ethical point of view, we would want to examine is the opinion of the board, the opinion of the executive director, and the opinion of the administrative assistant.

We are going to use the concept of an estimated value to demonstrate the perspectives of the individual stakeholders. We will use a decision tree to evaluate the overall ethical pursuits.

For each of our ethical values, one could assign a value, and think of it as a net ethical value. This of course is subjective. We will demonstrate this subjective value can be based on accepted ethical principles. This net ethical value could then be multiplied by the weight, the probability-like number, assigned to the ethical principle from the perspective of a stakeholder to give us an expected ethical value from each stakeholder. For our model to be more inclusive, in addition to our ethical principles, we contend one needs to address monetary concerns as an additional principle.

Terminating her, for example, could have a negative impact, not only on her and her family, but also on the community at large. People, in general, expect a long-standing organization to behave in a certain way; as such, it may have a negative impact on its reputation. At the same time, it could have a positive impact on the organization from a perspective of fiscal responsibility.

There are ethical principles which seem to guide toward keeping her employed. They might include compassion and kindness, respect for human dignity and loyalty. On the other hand, there are principles that encourageterminating her. These might include principles of justice and fairness and perhaps, universal principles such as the traditional work ethic-a day's pay for a day's work.

We need to consider the following perspectives. Utilitarianism is the doctrine that an ethical act is one which results in the greatest good (or the highest incidence of pleasure over pain) for the greatest number in the long run; thus, examining consequences, as one does when assessing the impact of a decision on the various stakeholders, is utilitarian based. One need not appeal to any principle other than the Principle of Utility. On the other hand, deontologists hold that an action's morality is completely independent of its consequences. "The deontological perspective views behavior as ethical or unethical by examining the rules and principles that guide behaviors and is based on a system of rights and duties. One's duty is to do what is morally right and to avoid what is morally wrong, regardless of the consequences" [5].

In an ideal world, we would have to find a way of evaluating ethical principles, not based on their consequences, but on some inherent hierarchical value. One would have to be able to say, for example, that as a rule compassion bears a higher ethical value than justice. Are certain values are deemed to be of higher value or greater importance than others? Can values be rank ordered? As previously mentioned, we allow for a subjective evaluation.

Allowing for values to ethical and practical principles, at this point we will define them as Kindness, Justice, and Finances. We will let the reader determine 


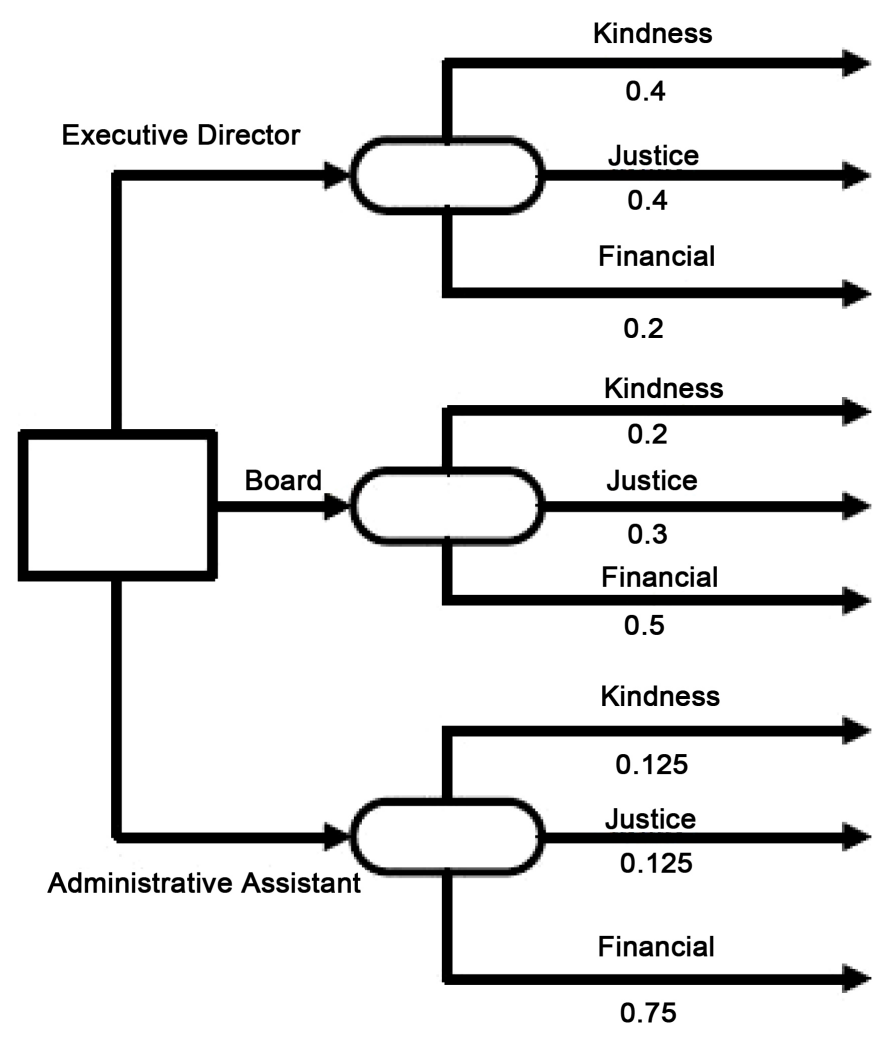

Figure 2. Example of an ethics decision tree [3].

the value of each. When we look at the Estimated Value (EV) from the perspective of the Executive Director, we may have something like the following (assuming the agreed values for the ethical principles):

EV of Executive Director $=2 / 5$ (Kindness) $+2 / 5$ (Justice) $+1 / 5$ (Finances), whereas the $\mathrm{EV}$ of the Administrative Assistant $=1 / 8$ (Kindness $)+1 / 8$ (justice $)+$ 3/4 (Finances).

Regarding using a decision tree as a tool, we would have different branches emanating from the root, each one representing an ethical principle or a principle under consideration from different stakeholders. Each branch would have the probability number (or its equivalent decimal) attached to it of a stakeholder. We would multiply these numbers along a given branch. Arriving at the leaf of a branch we would multiply the probabilities of each of the stake holders along the branch. Thus, at the end, we could compare the products at each of the leaves and see which ethical principle has the overall highest rating. Of course, this is under the assumption that each principle has a net ethical value. Figure 2 illustrates how a decision tree can be used in these circumstances.

Ultimately, we contend that we initiate our decisions by an ethical approach but in the end, we decide based on the results of our actions. This is analogous to a mathematical model where we accept certain hypotheses to generate the model; if the outcome of the model is not acceptable or realistic, then we go back and adjust the initial hypotheses. The ideas presented should serve as a model and have the benefit of raising these principles to a level of consciousness. 


\section{Conflicts of Interest}

The author declares no conflicts of interest regarding the publication of this paper.

\section{References}

[1] Aufmann, R., et al. (2018) Mathematical Excursions. Cengage Learning, Boston.

[2] Glazerman, A. (2021) Personal interview. 28 April 2021.

[3] Ben-Jacob, O. (2021) Personal interview. 5 May 2021.

[4] Glasser, D. (2005) To Learn and to Do. URJ Press, New York.

[5] Beu, D. and Buckley, M.R. (2001) The Hypothesized Relationship between Accountability and Ethical Behavior. Journal of Business Ethics, 34, 58. 